\title{
SÍNDROME DE TAKOTSUBO EN PACIENTE CON CARDIOPATÍA HIPERTRÓFICA
}

\author{
TAKOTSUBO SYNDROME IN PATIENT WITH HYPERTROPHIC \\ CARDIOMYOPATHY
}

FRANCO FACCIUTO' ${ }^{\prime}$ LEONARDO A. PILÓN' ${ }^{\prime}$, FRANCO L. MARINO', DANIELA F. CHILABERT ${ }^{2}$, FERNANDO BAGNERA ${ }^{3}$, GERARDO ZAPATA ${ }^{4}$

\begin{abstract}
RESUMEN
La cardiomiopatía de takotsubo (CT) es una condición aguda y reversible, caracterizada por una disfunción ventricular principalmente de los segmentos apicales del ventrículo izquierdo $(\mathrm{VI})$, con hipercontractilidad de los basales. Esto puede generar en ciertas ocasiones una obstrucción del tracto de salida del VI, lo que se puede exacerbar aún más en pacientes con cardiopatías hipertróficas. Debido a que la combinación de estas dos entidades es un escenario muy poco frecuente y a que su comportamiento en agudo muchas veces no es del todo benigno, su manejo puede ser extremadamente desafiante.

A continuación, se presenta el caso de un paciente de 72 años con hipertrofia ventricular izquierda y $\mathrm{CT}$ que evoluciona con shock cardiogénico.
\end{abstract}

Palabras clave: cardiomiopatía de takotsubo; cardiomiopatía hipertrófica; síndrome coronario agudo; shock cardiogénico.

\section{ABSTRACT}

Takotsubo cardiomyopathy (TCM) is an acute and reversible condition, characterized by ventricular dysfunction mainly of the apical segments of the left ventricle (LV), with hypercontractility of the basal cells. This can sometimes lead to obstruction of the LV outflow tract, which can be exacerbated even more in patients with hypertrophic heart disease. Because the combination of these two entities is a very rare scenario and because their behavior in the acute stage is often not entirely benign, their management can be extremely challenging.

Next, the case of a 72-year-old patient with left ventricular hypertrophy and TCM who progresses with cardiogenic shock is presented

Keywords: takotsubo cardiomyopathy; hypertrophic cardiomyopathy; acute coronary syndrome; cardiogenic shock.

REVISTA CONAREC 2018;33(146):255-258 | DOI:10.32407/RCON/2018146/0255-0258

\section{INTRODUCCIÓN}

La cardiomiopatía de takotsubo (CT), también conocida como síndrome de takotsubo, cardiomiopatía por estrés, síndrome de globo apical y síndrome del "corazón roto", es una condición clínica descrita por Sato en los años 90, caracterizada por una disfunción sistólica transitoria del ventrículo izquierdo (VI) que suele simular, al momento de presentarse, un infarto agudo de miocardio, con alteraciones electrocardiográficas en el segmento ST-T y discreto aumento de biomarcadores cardíacos ${ }^{1}$. Habitualmente es precedida por un desencadenante emocional o físico ocurriendo predominantemente en mujeres posmenopáusicas. Su patogenia no es conocida completamente aunque los mecanismos sugeridos en su génesis hacen referencia a mediación catecolaminérgica, espasmo coronario difuso, disfunción microvascular y miocardio aturdido neurogénico. El diagnóstico habitualmente es clínico, aunque en la mayoría de los casos se requiere una angiografía coronaria y una evaluación en serie de la fun-

1. Médico residente de Cardiología.

2. Jefe de Residentes de Cardiología

3. Médico Cardiólogo Staff de Unidad Coronaria.

4. Médico Cardiólogo Jefe de Unidad Coronaria. Instituto Cardiovascular de Rosario. Rosario, provincia de Santa Fe, Rep. Argentina.

$\triangle$ Correspondencia: Dr. Franco Facciuto. Bv Oroño 450, S2000DSR Rosario, Santa Fe, Rep.Argentina.facciutof@gmail.com

Los autores declaran no tener conflictos de intereses.

Recibido: 30/10/2018 | Aceptado: 10/11/2018 ción del $\mathrm{V}^{2,3}$. A pesar de que la recuperación completa de su función ocurre en 4 a 8 semanas, el riesgo de complicaciones intrahospitalarias graves como el shock cardiogénico (SC) es mayor en comparación con el infarto agudo de miocardio (11,4\% vs. $8 \%)$, evento que aumenta la mortalidad en 5 veces con respecto a aquellos sin SC 4,5 . Aunque este último puede ser secundario a disfunción sistólica, también puede ser causado por obstrucción del tracto de salida del VI (OTSVI), lo que se describió en el 10\% al 25\% de los pacientes con CT. Una posible hipótesis hace alusión a la marcada hiperdinamia de los segmentos basales, los cuales causarían un gradiente de presión intraventricular con un movimiento sistólico anterior de la valva mitral (MASVM), asociado a regurgitación mitral que conduciría a mayor colapso hemodinámico ${ }^{6}$.

La hipertrofia del VI es un fenómeno adaptativo muy frecuentemente diagnosticado en pacientes con hipertensión arterial de larga data y mal control. En dichas situaciones, adopta generalmente una distribución concéntrica y debe ser considerada una manifestación de daño de órgano blanco. En otras, puede estar relacionada con la presencia de trastornos genéticos (la mayoría autosómicos) conformando lo que se denomina miocardiopatía hipertrófica (MCH). Esta es la cardiopatía más frecuentemente relacionada a muerte súbita en los jóvenes y afecta en mayor medida al tabique ventricular (aunque la hipertrofia puede localizarse en cualquier región del VI). En dos tercios de los casos puede acompañarse de OTSVI "dinámica"7 y aún en pacientes sin gradiente en reposo puede generar una obstrucción "latente" desencadenada por factores adrenérgicos que alteran el estado hemodinámico. La ecocardiografía desempeña un papel importante en su diagnóstico: las imágenes bidimensionales son capaces 


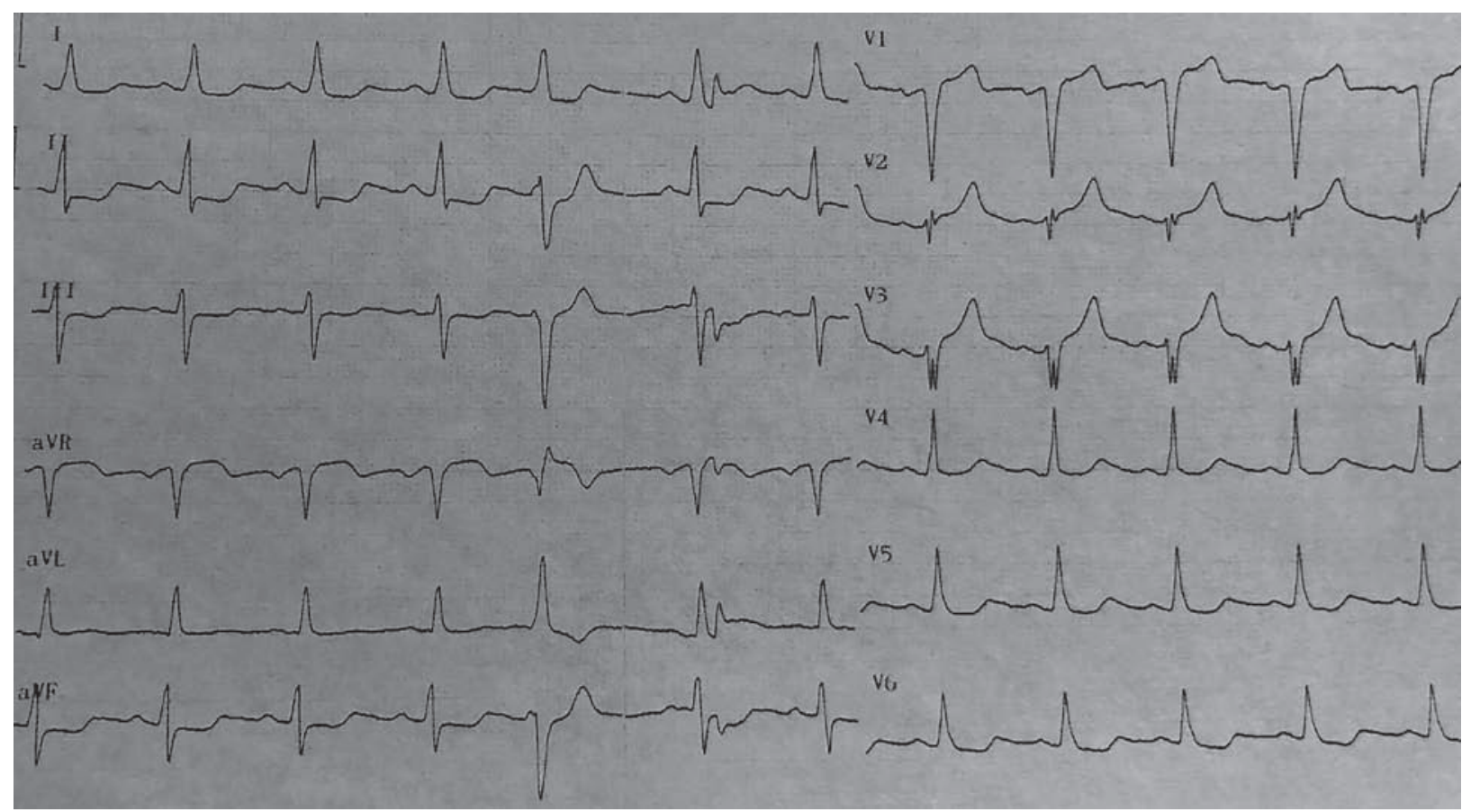

Figura 1. Electrocardiograma de ingreso: Se observa ritmo sinusal, con una frecuencia cardíaca de 75 latidos por minuto, supradesnivel del segmento ST de 6 milímetros de sumatoria de V1 a V3 e infradesnivel del segmento ST en DII, DIII y aVF.

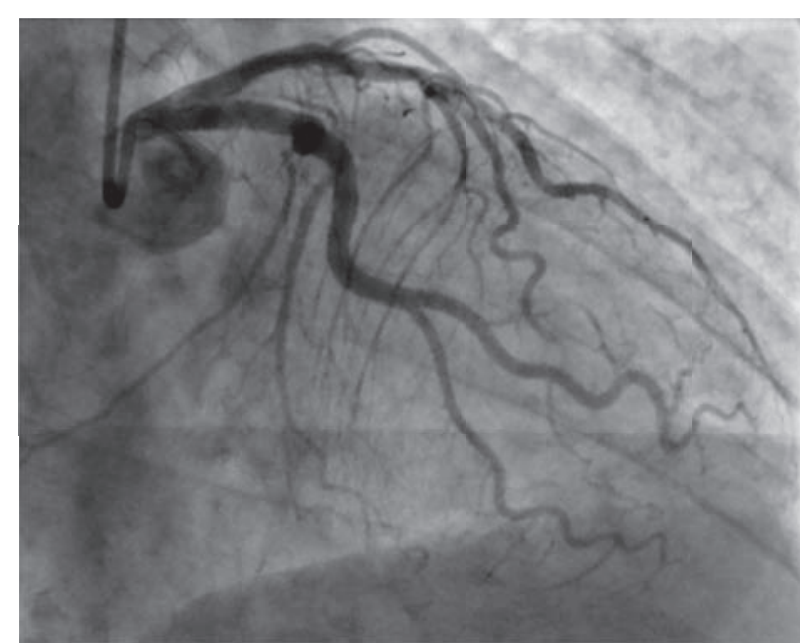

Figura 2. Cinecoronariografía que muestra ausencia de enfermedad coronaria epicárdica.

de cuantificar la magnitud de dicha hipertrofia, las vistas apicales de cuatro y cinco cámaras ayudan a identificar la gravedad y la duración del MASVM, y el Doppler color puede ayudar a localizar el nivel de obstrucción y a cuantificar su severidad ${ }^{8,9}$.

\section{CASO CLÍNICO}

Paciente de sexo masculino de 72 años, con hipertensión arterial en tratamiento con valsartán 160 mg y amlodipina 5 mg, sin antecedentes de enfermedad cardiovascular, que ingresó por dolor torácico de características opresivas de reposo, de 5 horas de evolución. Ante la sospecha etiológica de isquemia miocárdica aguda, se lo evaluó mediante electrocardiograma que evidenció supradesnivel del segmento ST de V1 a V3 con ondas Q en dicho terri- torio e infradesnivel inferolateral (Figura 1), por lo que se realizó cinecoronariografía de emergencia con el fin de adoptar eventual estrategia de reperfusión con angioplastia primaria pero la misma no mostró lesiones angiográficamente significativas (Figura 2), se realizó ventriculograma izquierdo que evidenció aquinesia apical con deterioro moderado a severo de la función sistólica del VI (Figura 3). Simultáneamente se solicitaron biomarcadores cardíacos, entre ellos troponina T de alta sensibilidad, que reflejaron valores marcadamente elevados (511,8 ug/l) por lo que se interpretó el cuadro como infarto agudo de miocardio con arterias coronarias epicárdicas angiográficamente normales y se inició tratamiento antiisquémico y antitrombótico. Se decidió continuar evaluación con ecocardiograma Doppler donde se encontró como datos positivos una marcada hipertrofia concéntrica del VI, con un septum y una pared posterior de 16 y $12 \mathrm{~mm}$, respectivamente, hipertrofia del septum basal (19 mm), una fracción de eyección del VI del 30\% con aquinesia apical en todos sus segmentos y del septum medio, y un gradiente medioventricular de reposo de 121 $\mathrm{mmHg}$ (Figura 4).

A las 8 horas de su estadía en unidad coronaria, evolucionó con hipotensión arterial sostenida y rales crepitantes en ambos campos pulmonares con radiografía de tórax con signos de redistribución de flujo, interpretándose el cuadro como SC. Se inició tratamiento con furosemida e inotrópicos (dobutamina y noradrenalina) durante 7 días, con buena respuesta clínica.

El paciente fue externado a los 13 días de su ingreso, con un electrocardiograma que mostraba negativización de ondas T en DI, aVL y de V3 a V6, previa evaluación con SPECT cardíaca de reposo para evaluar tamaño de infarto con perfusión normal en todos sus segmentos (tamaño de infarto de $0 \%$, fracción de eyección de $58 \%$ y volúmenes ventriculares normales) (Figura 5). 


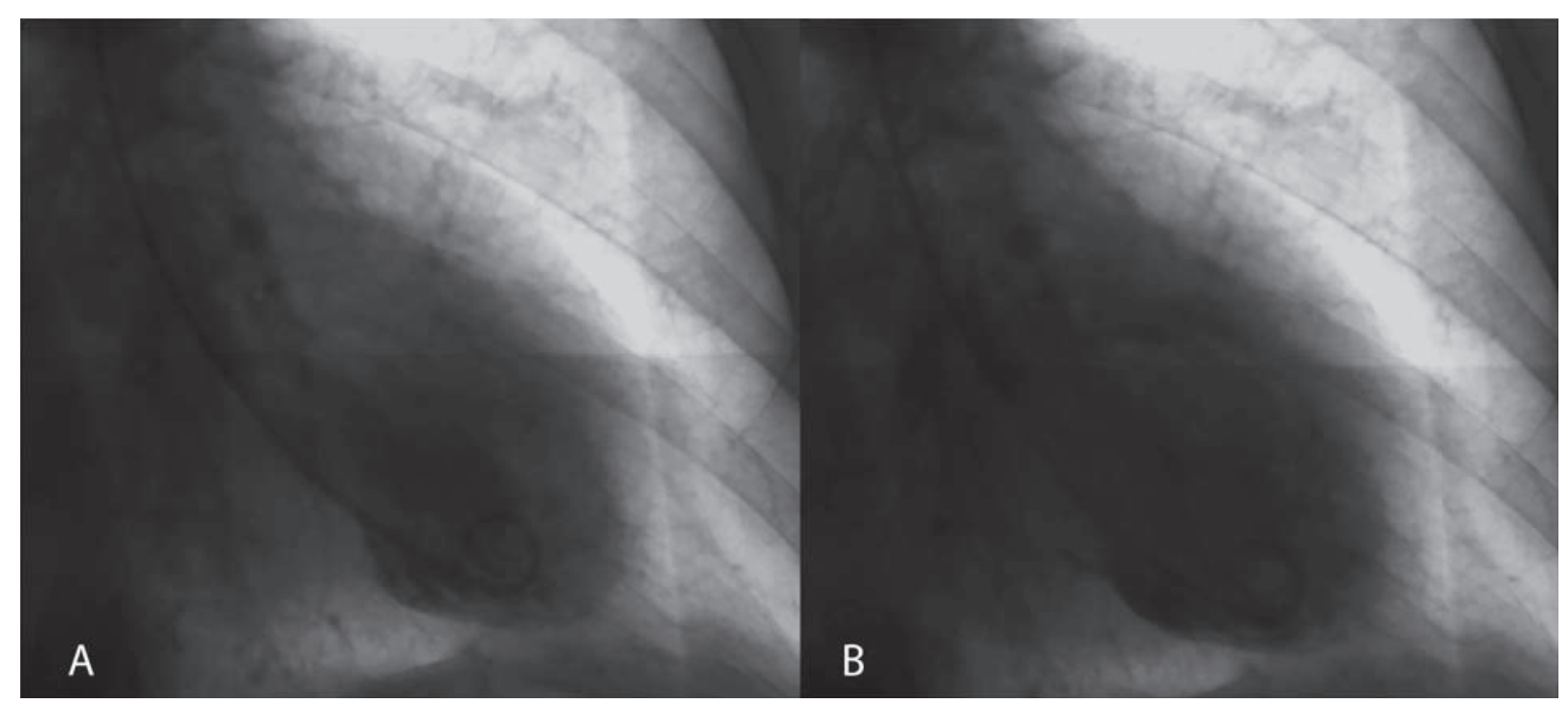

Figura 3. Ventriculograma izquierdo en sístole (A) y diástole (B) que muestra aquinesia apical con deterioro moderado a severo de la función sistólica del ventrículo izquierdo.

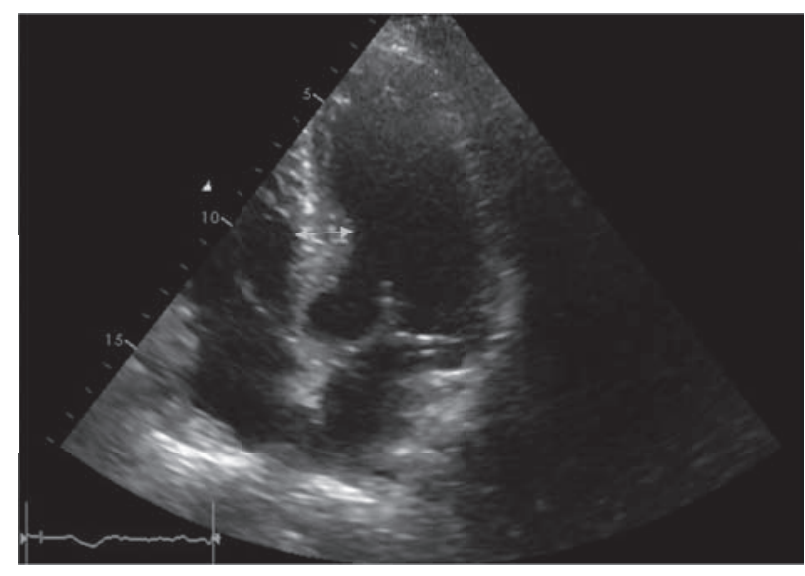

Figura 4. Ecocardiograma. Se muestra una vista apical de cuatro cámaras con hipertrofia septal (doble flecha).

\section{DISCUSIÓN}

Debido a su baja prevalencia, no es frecuente el reporte en la bibliografía de casos clínicos que combinen la presencia de CT en pacientes con MCH. En algunos la CT ocurrió en pacientes previamente diagnosticados con $\mathrm{MCH}^{10-13}$ y en otros la $\mathrm{MCH}$ no fue diagnosticada y la CT fue la causa de su enmascaramiento ${ }^{14-16}$.

Es clara la mayor prevalencia de CT en mujeres, aunque el sexo no descarta su diagnóstico, y en este caso no se identificó un factor estresante físico o emocional. Muchas veces los mismos pacientes minimizan estas cuestiones y tal vez no se pesquisó dicho dato en la anamnesis.

En el caso del paciente previamente presentado, tenía como antecedente a la hipertensión arterial, por lo que puede que se trate de una cardiopatía hipertensiva. Es llamativa su magnitud y disposición a nivel del septum basal y la presencia de gradiente medioventricular, datos raramente encontrados en cardiopatías hipertensivas, por lo que no podemos descartar que se trate de una MCH con hipertensión arterial asociada, aunque claro está que esta última serviría de factor

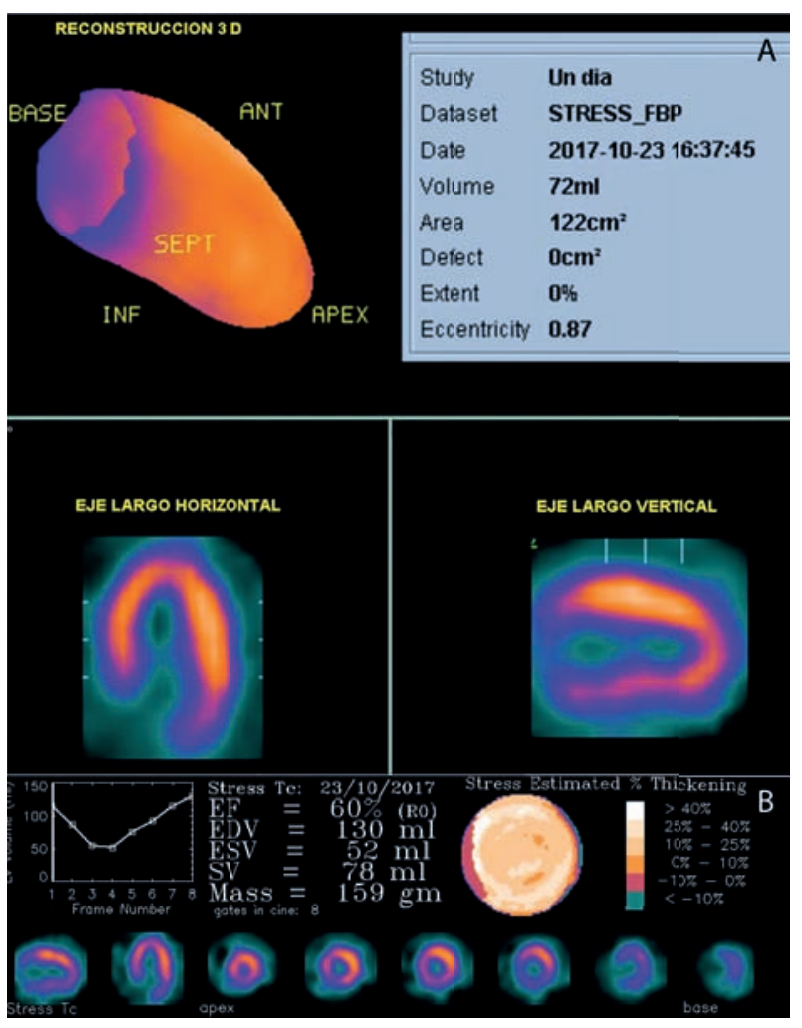

Figura 5. SPECT cardíaca de reposo. A. Normoperfusión en todos los segmentos. Tamaño infarto: $0 \%$. B. Fracción de eyección del ventrículo izquierdo conservada, con volúmenes ventriculares en límites normales.culares en límites normales.

para excluir a la primera ya que se la considera causa identificable de hipertrofia ventricular. Otra entidad descrita es el septum sigmoideo, también reconocido como hipertrofia septal localizada, nudillo o protuberancia septal. Sus causas y consecuencias son controvertidas; algunos lo consideran parte del espectro fenotípico de la $\mathrm{MCH}$, pero la mayoría, una entidad diferenciada y relacionada principalmente con una mayor edad, mayor angulación del septum interventricular respecto de la aorta o a hipertensión arterial. Clínicamente sue- 
le ser asintomático pero podría causar disnea de esfuerzo por OTSVI en algunas situaciones ${ }^{17}$. De igual manera, independientemente de la etiología, el manejo del paciente portador de esta condición no difiere y su complejidad sigue siendo sustancial.

La presencia de hipotensión en pacientes con CT debe evaluarse cuidadosamente con el fin de diferenciar bajo gasto secundario a disfunción ventricular izquierda del generado por OTSVI, ya que el uso de inotrópicos comunes puede empeorar el cuadro en este último caso. Aunque es un escenario muy poco frecuente, su tratamiento es extremadamente desafiante. La reanimación con líquidos (en ausencia de congestión pulmonar significativa) y los betabloqueantes son las opciones terapéuticas iniciales. Estas no fueron estrategias que pudimos aplicar ya que el paciente se encontraba claramente con signos de insuficiencia cardíaca retrógrada y anterógrada. Los agonistas alfa se pueden agregar para aumentar la presión arterial, lo que ayuda a disminuir el gradiente al aumentar la poscarga? .

\section{BIBLIOGRAFÍA}

1. Sato H, Tateishi H, Uchida T, et al. Takotsubo type cardiomyopathy due to multivessel spasm. In: Clinical aspect of myocardial injury: from ischemia to heart failure. Tokyo: Kagaku Hyoronsha 1990;56-64.

2. Ghadri J-R, Wittstein IS, Prasad A, Sharkey S, Dote K, Akashi YJ, et al. International Expert Consensus Document on Takotsubo Syndrome (Part 1): Clinical Characteristics, Diagnostic Criteria, and Pathophysiology. Eur Heart J 2018;39(22):2032-46.

3. Akashi YJ, Goldstein DS, Barbaro G, Ueyama T. Takotsubo cardiomyopathy: a new form of acute, reversible heart failure. Circulation 2008;118(25):2754-62.

4. Templin C, Ghadri JR, Diekmann J, Napp LC, Bataiosu DR, Jaguszewski M, et al. Clinical features and outcomes of Takotsubo (stress) cardiomyopathy. N Engl J Med 2015;373(10):929-938.

5. Almedro-Delia M, Núñez-Gil IJ, Lobo M, Andrés M, Vedia O, Sionis A, et al. Shortand Long-Term prognostic relevance of cardiogenic shock in Takotsubo Syndrome: Results from the RETAKO Registry. JACC Heart Fail 2018;6(11):928-36.

6. Fefer P, Chelvanathan A, Dick AJ, Teitelbaum EJ, Strauss BH, Cohen EA. Takotsubo cardiomyopathy and left ventricular outflow tract obstruction. J Interv Cardiol 2009;22(5):444-52

7. Elliott PM, Anastasakis A, Borger MA, Borggrefe M, Cecchi F, Charron P, et al. Guía de práctica clínica de la ESC 2014 sobre el diagnóstico y manejo de la miocardiopatía hipertrófica. Rev Esp Cardiol 2015;68(1):63.e1-e52.

8. Chockalingam A, Tejwani L, Aggarwal K, Dellsperger KC. Dynamic left ventricular outflow tract obstruction in acute myocardial infarction with shock: cause, effect, and coincidence. Circulation 2007;116(5):e110-3.

9. Akita K, Maekawa Y, Tsuruta H, Okuda S, Yanagisawa R, Kageyama T, et al. "Moving left ventricular obstruction" due to stress cardiomyopathy in a patient with hy-
Nuestro paciente complicó con SC que se trató con éxito con la administración gradual de distintos inotrópicos bajo un control hemodinámico cercano, y resolvió luego de nueve días sin requerir la colocación de dispositivos de asistencia ventricular.

Previo al alta se le solicitó una SPECT cardíaca de reposo que descartó áreas de fibrosis, avalando la fisiopatología planteada: un importante atontamiento miocárdico que resuelve a las pocas semanas.

\section{CONCLUSIÓN}

La combinación de cardiomiopatía de takotsubo y cardiopatía hipertrófica no es frecuentemente descripta por la bibliografía, por lo que conocer su expresión clínica resulta de gran interés en la práctica diaria teniendo en cuenta la complejidad de su manejo. pertrophic obstructive cardiomyopathy treated with percutaneous transluminal septal myocardial ablation. Int J Cardiol 2016;202:194-5.

10. Ochiumi Y, Ikeda S, Hamada M. Reappearance of the left ventricular pressure gradient in a patient with hypertrophic obstructive cardiomyopathy. Intern Med 2015;54(7):805-6.

11. Singh NK, Rehman A, Hansalia SJ. Transient apical ballooning in hypertrophic obstructive cardiomyopathy. Tex Heart Inst J 2008;35(4):483-4.

12. Jaber WA, Wright SR, Murphy J. A patient with hypertrophic obstructive cardiomyopathy presenting with left ventricular apical ballooning syndrome. J Invasive Cardiol 2006;18(10):510-2.

13. Patrianakos AP, Nyktari E, Parthenakis FI, Vardas PE. Reversible left ventricular apical ballooning after heavy alcohol consumption in a patient with hypertrophic cardiomyopathy. Int J Cardiol 2013;164(3):e29-31.

14. Roy RR, Hakim FA, Hurst RT, Simper D, Appleton CP. Two cases of apical ballooning syndrome masking apical hypertrophic cardiomyopathy. Tex Heart Inst $J$ 2014;41(2):179-83.

15. Daralammori Y, El Garhy M, Gayed MR, Farah A, Lauer B, Secknus MA. Hypertrophic obstructive cardiomyopathy masked by Tako-tsubo syndrome: a case re port. Case Rep Cardiol 2012;2012:486427.

16. Brabham WW, Lewis GF, Bonnema DD, Nielsen CD, O'Brien TX. Takotsubo cardiomyopathy in a patient with previously undiagnosed hypertrophic cardiomyopathy with obstruction. Cardiovasc Revasc Med 2011;12(1):70.e1-5.

17. Gentille-Lorente D, Salvadó-Usach T. Septo sigmoideo: ¿Una variante de la hipertrofia ventricular o de la miocardiopatía hipertrófica?. Arch Cardiol Mex 2016;86(2):110-122. 